\title{
Evaluation Of Risk Factors And Epidemiological Study In Myocardial Infarction Patients In Tertiary Care Hospital
}

\author{
Anju Sharma*, Konda. Sushritha*, Konda. Suman ${ }^{* *}$, Palakurthy. Harish Goud ${ }^{* *}$, Sadanandamn Akari** \\ *Department of pharmacy practice, PHARM D, \\ ** Mallareddy institute of pharmaceutical sciences, Dulapally, medchal, Telangana, India. \\ DOI: 10.29322/IJSRP.10.06.2020.p10286 \\ http://dx.doi.org/10.29322/IJSRP.10.06.2020.p10286
}

\begin{abstract}
Background and objectives: Recent ecological study for major CVD risk factors and mortality indicates a high correlation between expected and observed mortality rates for three major risk factors- hypertension, diabetes, and smoking. Survivor of MI are at higher risk of recurrent infarction than in people who don't have coronary heart disease.
\end{abstract}

Objective: To evaluate the risk factors of Myocardial Infarction among cardiac patients and provide patient education in order to minimize risk factors. To provide patient education and lifestyle modifications in order to minimize myocardial infarction patients.

Patients and method: A prospective and observational study was conducted on 500 medical records of data was collected by using a case report form for six months from August 2019 to February 2020 and was conducted in the cardiac department of Malla-Reddy Narayana Multi-Specialty Hospital.

Result: We have assessed around 500 myocardial infarction cases, Out of which higher proportion of risk factors in myocardial infarction patients has been observed in Coronary Artery Disease(31\%), Hypertension(20.9\%), followed by Diabetes Mellitus(15.01\%), Smoking(12.5\%), Alcohol consumption $(12.3 \%)$, Obesity $(3.09 \%)$ and Any co-existing diseases $(4.75 \%)$. Males $(72.4 \%)$ are more effected than females $(27.6 \%)$.

Conclusion: On the whole, this study indicates that evaluation of risk factors provides new scope for the development of more effective approaches to prevent the recurrent chances of myocardial infarction by providing patient education and life style modification.

Index Terms- Hypertension; Myocardial Infarction; Patient education; Risk factors.

\section{INTRODUCTION}

$\mathrm{W}$ Epidemiological study on myocardial infarction:

$\mathrm{HO}$ and Global burden of disease study mainly focuses on increasing trends in the years of life lost and disability adjusted life years from coronary heart disease in India.

In India, studies have reported aggravating Coronary Heart Disease (CHD) prevalence over past 60years from $<1 \%$ to $4 \%-6 \%$ in rural population and $1 \%$ to $9 \%-10 \%$ in urban population ${ }^{1}$. The most popular form of coronary heart disease is myocardial infarction and is responsible for over $15 \%$ of mortality each year, among vast majority of people undergo Non-ST Elevated Myocardial Infarction (NSTEMI) than ST-Elevated Myocardial Infarction (STEMI). The prevalence of myocardial infarction is extreme in men in all specific age groups than women ${ }^{2}$.

By the end of $20^{\text {th }}$ century, Cardio Vascular Diseases (CVD) accounted for $<10 \%$ of all deaths worldwide. At this end, CVD accounted for nearly $50 \%$ of the deaths in the developed world and $25 \%$ of the deaths in developing world. By 2020, CVD may claim 25 million deaths annually. As this trend spreads to and continues in developing countries, CVD will dominate as major cause of death by 2020, accounting for at least 1 in every 3 death. Two main factors have been attributed to reduce in CVD mortality rates can be therapeutic advances and preventive measures. According to WHO, number of persons with diabetes mellitus varies from 135 million people in 1995 to 300million in 2025, where a $35 \%$ raise in worldwide prevalence is seen ${ }^{3}$.

Statistics on evaluation of risk factors: The funds from US and international studies shows a strong protective association between ideal $\mathrm{CV}$ health matrices and clinical, preclinical conditions. Tobacco use can be a leading cause and is estimated to account for 7.2 million deaths worldwide in 2015 and over past 5 years, there has been sharp increase in cigarette use among adolescents.

Coronary heart disease $(43.8 \%)$ is leading cause of death attributable to CVD in US, followed by stroke (16.8 \%), hypertension (9.4\%), heart failure (9\%) and other CVD'S (17.9\%) 4. Among children from 1999 to 2000, 2000 to 2015, prevalence of non-smoking, ideal total cholesterol and ideal blood pressure has been improved. The prevalence of current smoking in US in 2016 was $18.5 \%$ for adults, $3.4 \%$ of adolescents smoked cigarette in past month. According to NHANES i.e; National Health and Nutrition Exam Survey in 2015-2016 is 39.6\% of US adults and $18.5 \%$ of youth were obese and $87.7 \%$ of adults and $5.6 \%$ of youth had severe obesity. So, on basis of NHANES 2013-2016 data for adults with diabetes mellitus, $20.9 \%$ had their diabetes mellitus treated but uncontrolled, $9.2 \%$ were aware they had diabetes mellitus but were not treated ${ }^{5}$.

A better understanding of the risk factors associated Myocardial Infarction disease would enable the development of prevention strategies. No previous study, to our knowledge, has reported the Common risk factors associated with incidence of 
myocardial infarction. We therefore sought to identify risk factors for MI, with a particular emphasis on characteristics that are known to be Modifiable.

\section{MATERIALS AND METHODS:}

The study was conducted in the department of cardiology in Malla Reddy Narayana Multi-specialty Hospital, Hyderabad, India and the patients were enrolled in the health survey from $1^{\text {st }}$ July, 2019 to $31^{\text {st }}$ January, 2020. A total of 500 patients were involved in the study where 362 men and 138 women older than 30 years has been evaluated for risk factors of myocardial infarction. Patients were excluded from the current study if patient is pediatric, if the patient is pregnant or lactating, if the patient is suffering from drug induced myocardial infarction, if the patient is using recreational drugs (amphetamines, cocaine, LSD). The patients have agreed to participate in the baseline health examination which indicates written informed consent.

\section{Demographic details:}

Age and sex were self-reported by the patients. Height and Weight was directly measured using a calibrated scale. Blood pressure was measured using a manual sphygmomanometer where the patient was seated in a comfortable position.

\section{ECG reports:}

Conduction disorders such as lateral wall infarct, anterior wall infarct, inferior wall infarct, posterior wall infarct were manually confirmed for the purposes of the present study.

\section{Laboratory measurements:}

Blood samples were collected to monitor the levels of serum creatinine, troponin-1, CKMB, CPK, LDH, and plasma glucose using standard laboratory techniques.

\section{Cardiovascular comorbidities:}

Hypertension is observed when the systolic blood pressure is greater than $140 \mathrm{~mm} \mathrm{Hg}$, diastolic blood pressure is greater than $90 \mathrm{~mm} \mathrm{Hg}$, or any past history of hypertension with the usage of anti hypertensive medication. In the same way, Diabetes is observed when a fasting glucose level is of at least $200 \mathrm{mg} / \mathrm{dL}$ in a single measurement or at least $120 \mathrm{mg} / \mathrm{dL}$ in 2 separate measurements or usage of any anti diabetic medication. Myocardial infarction is diagnosed when any two of clinical features are present: a) ischemic chest pain, b)any new abnormal $\mathrm{Q}$ waves or ST segment changes c)abnormal elevation in cardiac enzymes.

\section{Habits:}

Alcohol consumption and cigarette smoking were evaluated by using questionnaires. Patients were classified as occasional smokers, regular smokers, or nonsmokers. Patients were also classified based on their amount of alcohol intake as chronic alcoholic, ex-alcoholic or non alcoholic.

\section{RESULTS:}

A total of 500 cases were examined for evaluation of risk factors in myocardial infarction patients during the study period, of which 500 cases were enrolled based on Inclusion and Exclusion criteria. There were 138 female patients and 362 male patients enrolled for the study period (Table1). Of the enrolled 500 patients, recording the age wise distribution of subjects with class size of 10 years, it was seen that majority of subjects belong to age group of 51-60 years, with mean age value of 50.54 years (Table 2). Among different types of MI, the most common types are NSTEMI and STEMI where 366 subjects have STEMI and 134 subjects have NSTEMI (Table 3). Of 500 subjects enrolled in this study, the length of hospitalization was evaluated where most of the subjects (375 cases) stay for 1-3 days followed by 125 cases for 4-6 days (Table 4). On the whole, this study mainly focuses on evaluation of few easily measured, preventable risk factors like smoking, alcohol, obesity where higher proportion of risk factors in myocardial infarction patients has been observed in Coronary Artery Disease $(31 \%)$, Hypertension $(20.9 \%)$, followed by Diabetes Mellitus(15.01\%), Smoking(12.5\%), Alcohol consumption(12.3\%), Obesity (3.09\%) and Any co-existing diseases $(4.75 \%$ ) (Table 5).

The data is analyzed using SPSS version 20. Study protocol was prepared and submitted to Human Ethical Committee of Malla Reddy Narayana Multi-specialty Hospital and it has been approved by the Ethics Committee.

\section{DISCUSSION:}

In this present scenario, there has been no proper evidence which demonstrate the proportion of risk factors among myocardial infarction patients where the reoccurrence chances are higher. Several recent studies on this strategy were retrospective or case-control analysis.

A population based cohort study was conducted by TuomasKerola, on risk factors associated with MI who concludes that the average age of 6146 subjects was 49.2years of which 2697 $(43.9 \%)$ were men and $3449(56.1 \%)$ were women ${ }^{6}$, where our study was carried out on evaluation of risk factors among myocardial infarction inpatients in department of coronary care unit(CCU) in territory care hospital.

There is a need of providing a proper patient education regarding disease and its associated risk factors in order to minimize the recurrent chances of MI. Hence the results also highlight several areas that need improvements. Overall, scope for minimizing risk factor has to be existed. When the patients case sheets were screened thoroughly, it was seen that majority of subjects belong to age group of 51-60 years, with mean age value of 50.54 years of which $138(27.6 \%)$ were female patients and $362(72.4 \%)$ were male patients.

Joshua Chadwick Jay raj et.al, study concludes that the most popular form of coronary heart disease is myocardial infarction and is responsible for over $15 \%$ of mortality each year, among vast majority of people undergo Non-ST Elevated Myocardial Infarction (NSTEMI) than ST-Elevated Myocardial Infarction (STEMI) ${ }^{2}$ where as in our study, we found that most of the cardiac patients were more likely affected with STEMI of 
around 366 subjects (73.2\%) when compared to NSTEMI i.e; 134 subjects $(26.8 \%)$.

Out of 500 subjects included in the study, 166(12.5\%) subjects had a social habit of smoking where few are chronic smokers and few are ex-smokers and this report was comparable to Emily Banks et.al study, but higher compared to report from Australia during 2006 to 2009 . However, Current tobacco smokers have at least double the chances of developing MI. Risks of MI increases with increase in number of cigarettes smoked per day and were greatly diminished among those quitting smoking ${ }^{7}$. MI is a serious result of Coronary Artery Disease (CAD) which is seen in higher proportion of $31 \%$. Out of 500 subjects, 415 were exposed to this risk factor and the recurrent chances are higher.

The current prospective observational study was carried to evaluate the risk factors of MI patients who are admitted in Coronary Care Unit (CCU) of tertiary care settings. Among different types of modifiable and non-modifiable risk factors, we have analyzed only specific risk factors which are the major causes for MI such as Hypertension, Diabetes Mellitus, Smoking, Alcohol, any co-existing CVDs, Obesity, and other comorbid conditions. Smoking and Alcohol are preventable risk factors for this disease. Overweight and obese may affect health, and it is necessary to control one's BMI.

Literature was evaluated only on specific individual risk factors, but very small amount in-detail conclusions of such studies were reported by other investigators. In the following study site, it was seen that the highest number of subjects were enrolled for the study belongs to cardiac department. Considering various risk factors, the highest proportion of Coronary Artery Disease was observed followed by hypertension and diabetes.

Providing patient education can also help in minimizing the risk factors, thereby reducing the incidence of myocardial infarction. Based on this study, in the following study period it can be concluded that improving the life style of individual can reduce the recurrent chances of myocardial infarction.

Tables and Figures:

Table I: Gender-wise distribution of patients

\begin{tabular}{|l|l|l|}
\hline Gender & Number of cases & Percentage \\
\hline Female & 138 & \\
\hline & & $27.6 \%$ \\
\hline Male & 362 & $72.4 \%$ \\
\hline Total & & \\
\hline
\end{tabular}

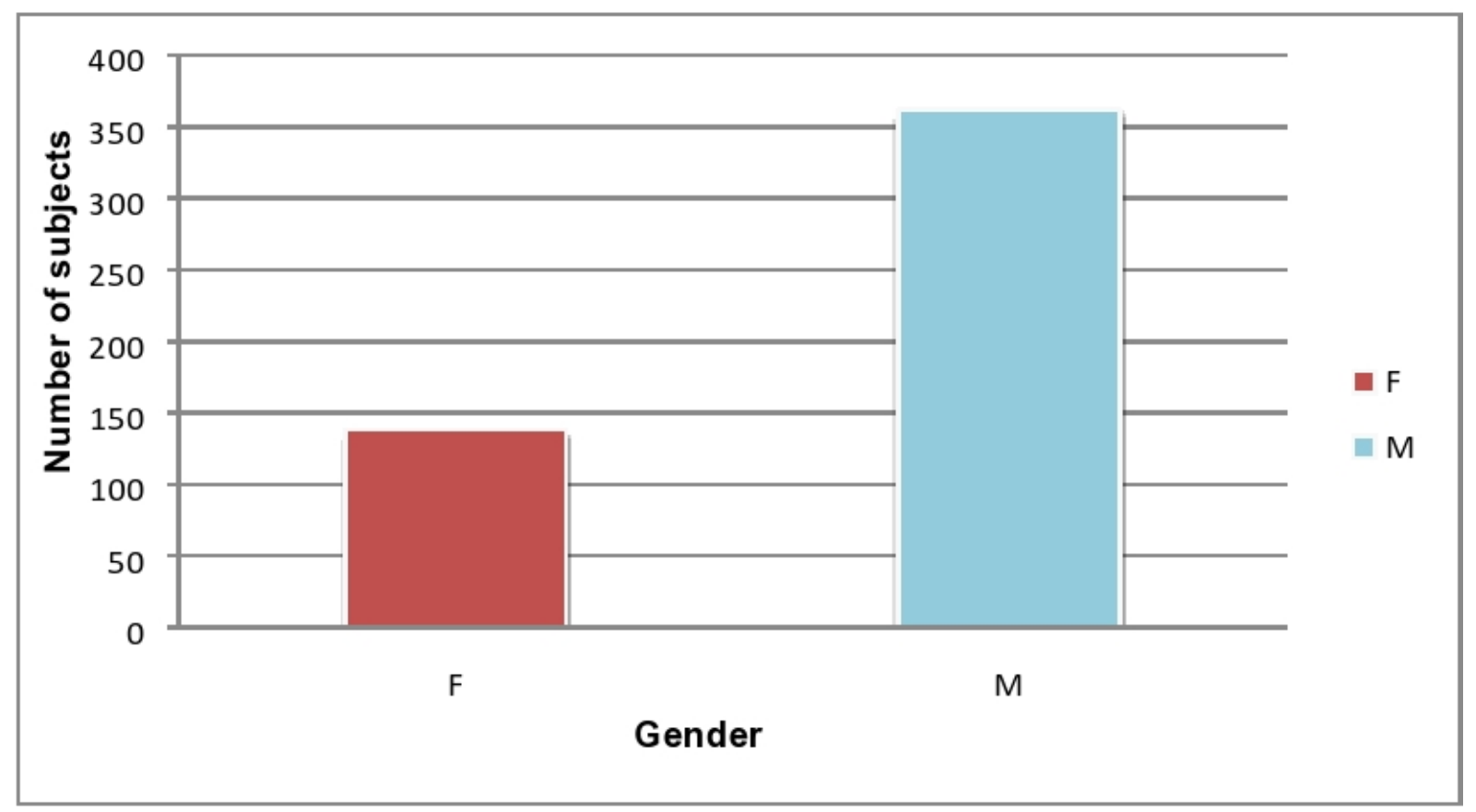

Figure 1: Graphical representation of Gender-wise Distribution 
Table II: Age-wise distribution of subjects

\begin{tabular}{|l|l|l|}
\hline AGE & No of Cases & Percentage \\
\hline $21-30$ & 3 & $0.6 \%$ \\
\hline $31-40$ & 51 & $10.2 \%$ \\
\hline $41-50$ & 129 & $25.8 \%$ \\
\hline $51-60$ & 151 & $30.2 \%$ \\
\hline $61-70$ & 120 & $24 \%$ \\
\hline $71-80$ & 41 & $8.2 \%$ \\
\hline $81-90$ & 5 & $1 \%$ \\
\hline Total & 500 & $100 \%$ \\
\hline & & \\
\hline
\end{tabular}

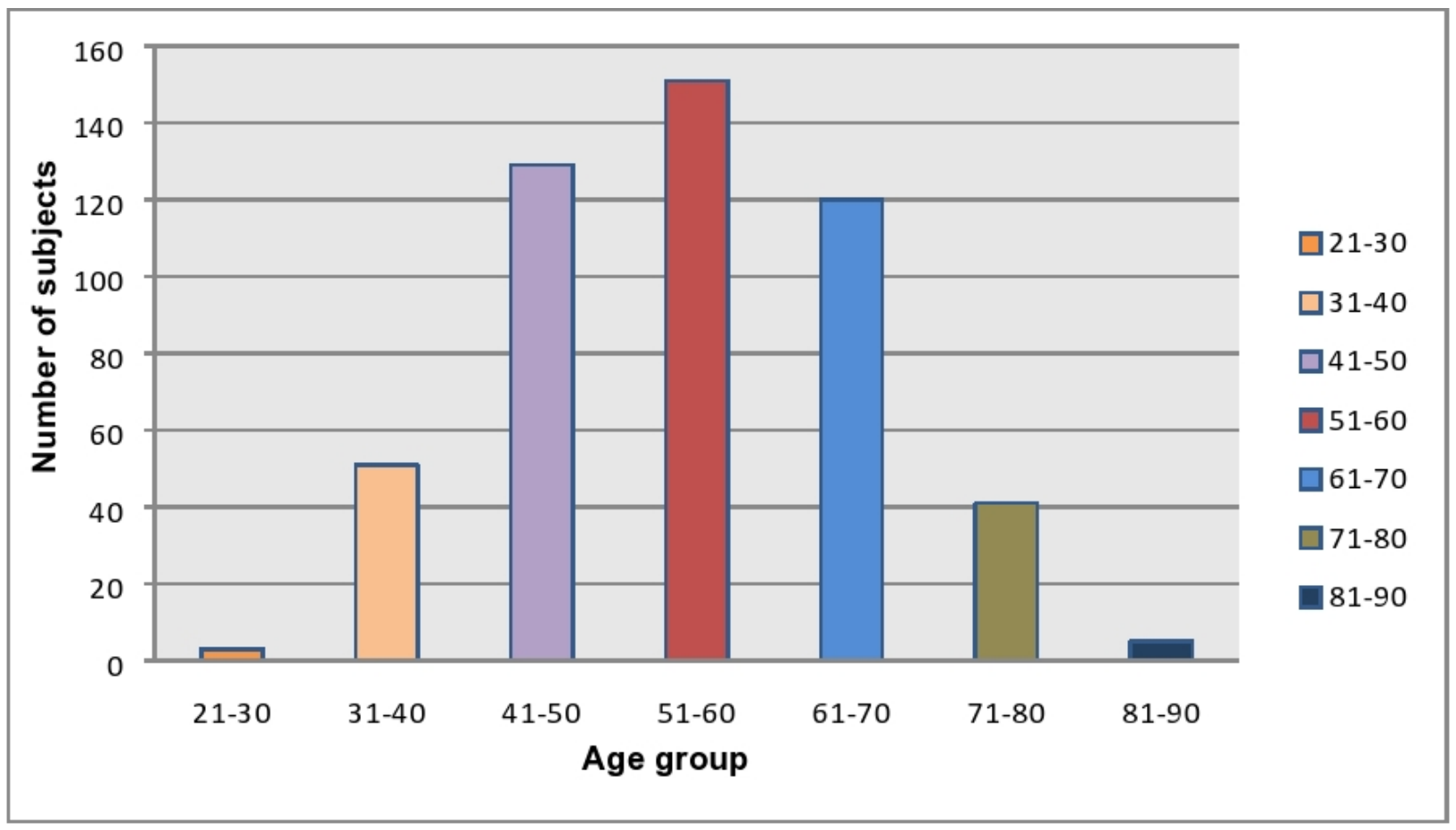

Figure 2: Graphical representation of age- wise distribution.

Table III: Type of myocardial infarction

\begin{tabular}{|l|l|l|}
\hline Type of MI & No Of cases & Percentage \\
\hline NSTEMI & 134 & 26.8 \\
\hline STEMI & 366 & 73.2 \\
\hline Total & 500 & 100 \\
\hline
\end{tabular}




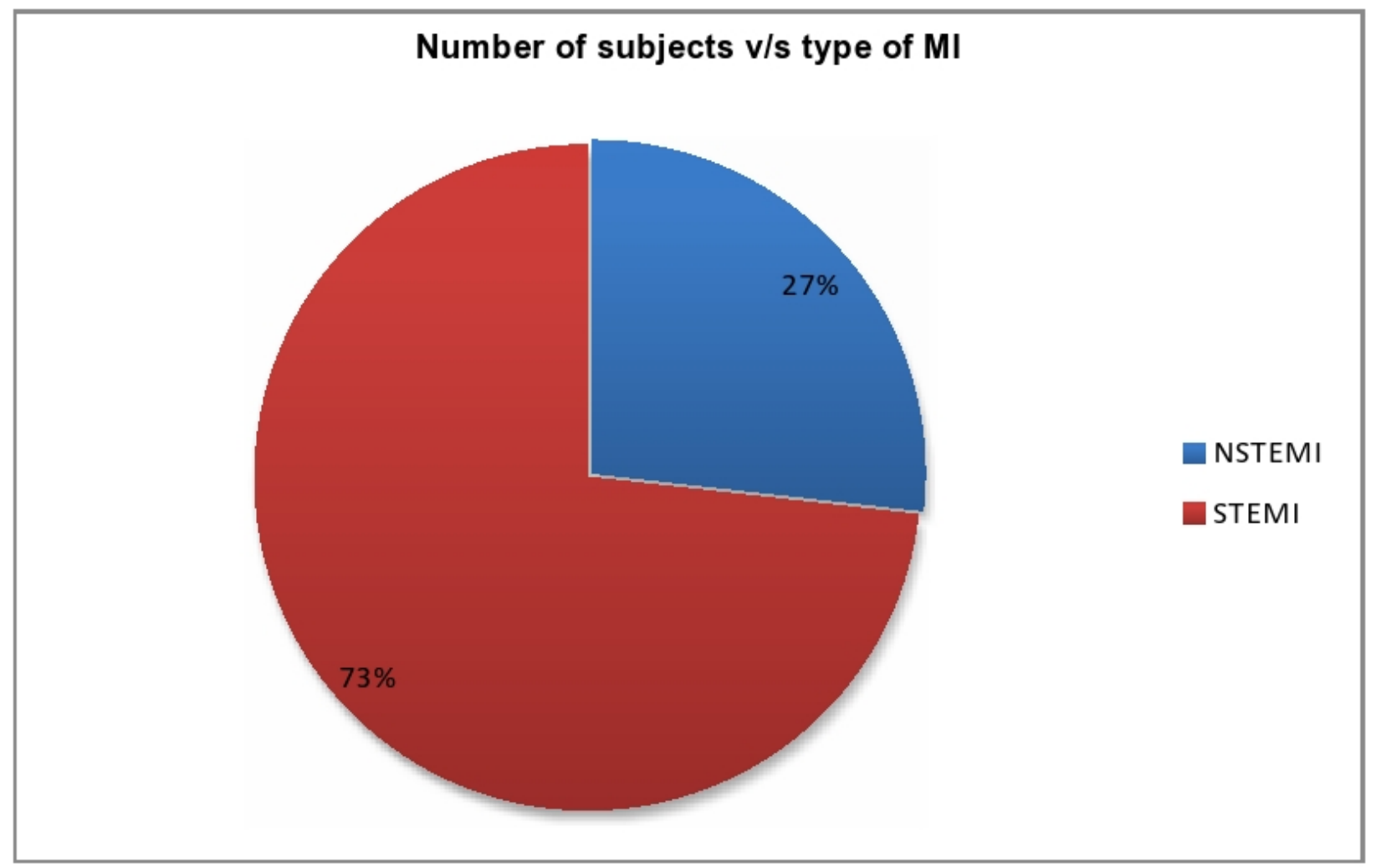

Figure 3: Pictorial representation of type of myocardial infarction among enrolled patients

Table IV: length of stay among enrolled subjects

\begin{tabular}{|l|l|l|}
\hline length of stay & number of cases & Percentage \\
\hline $1-3$ days & 275 & 60 \\
\hline $4-6$ days & 125 & 25 \\
\hline $7-9$ days & 65 & 8 \\
\hline $10-12$ days & 25 & 5 \\
\hline $13-15$ days & 10 & 2 \\
\hline
\end{tabular}




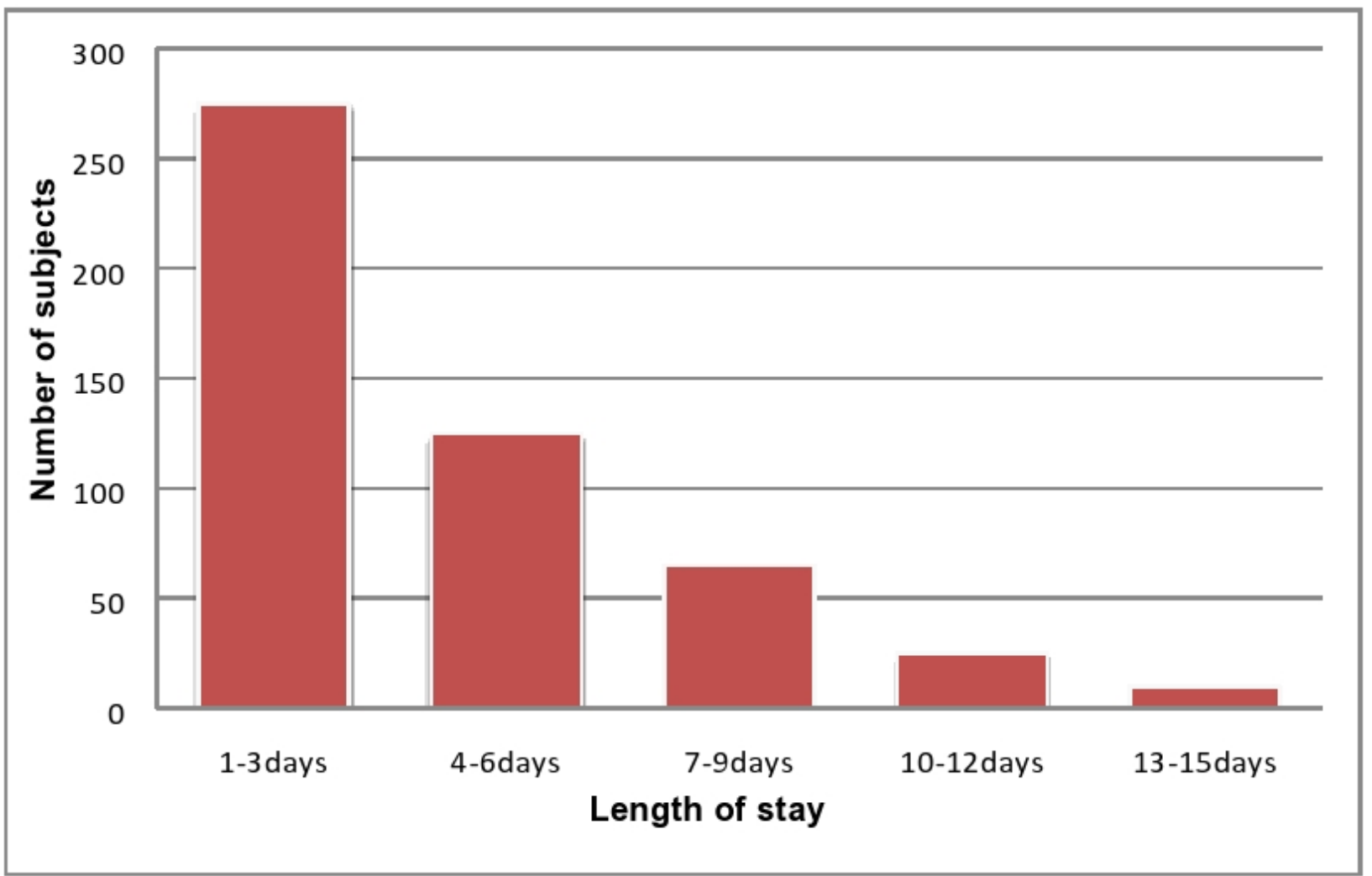

Figure 4: Graphical representation of length of stay among enrolled patients

Table V: Risk factors v/s number of subjects

\begin{tabular}{|l|l|l|}
\hline RISK FACTOR & APPERED IN NUMBER OF CASES & Percentage \\
\hline CAD & 415 & 31.32075472 \\
\hline OTHER & 63 & 4.754716981 \\
\hline SMOKING & 166 & 12.52830189 \\
\hline ALCOHOLIC & 164 & 12.37735849 \\
\hline HTN & 277 & 20.90566038 \\
\hline DM & 199 & 15.01886792 \\
\hline Obesity & 41 & 3.094339623 \\
\hline Total & 1325 & 100 \\
\hline
\end{tabular}




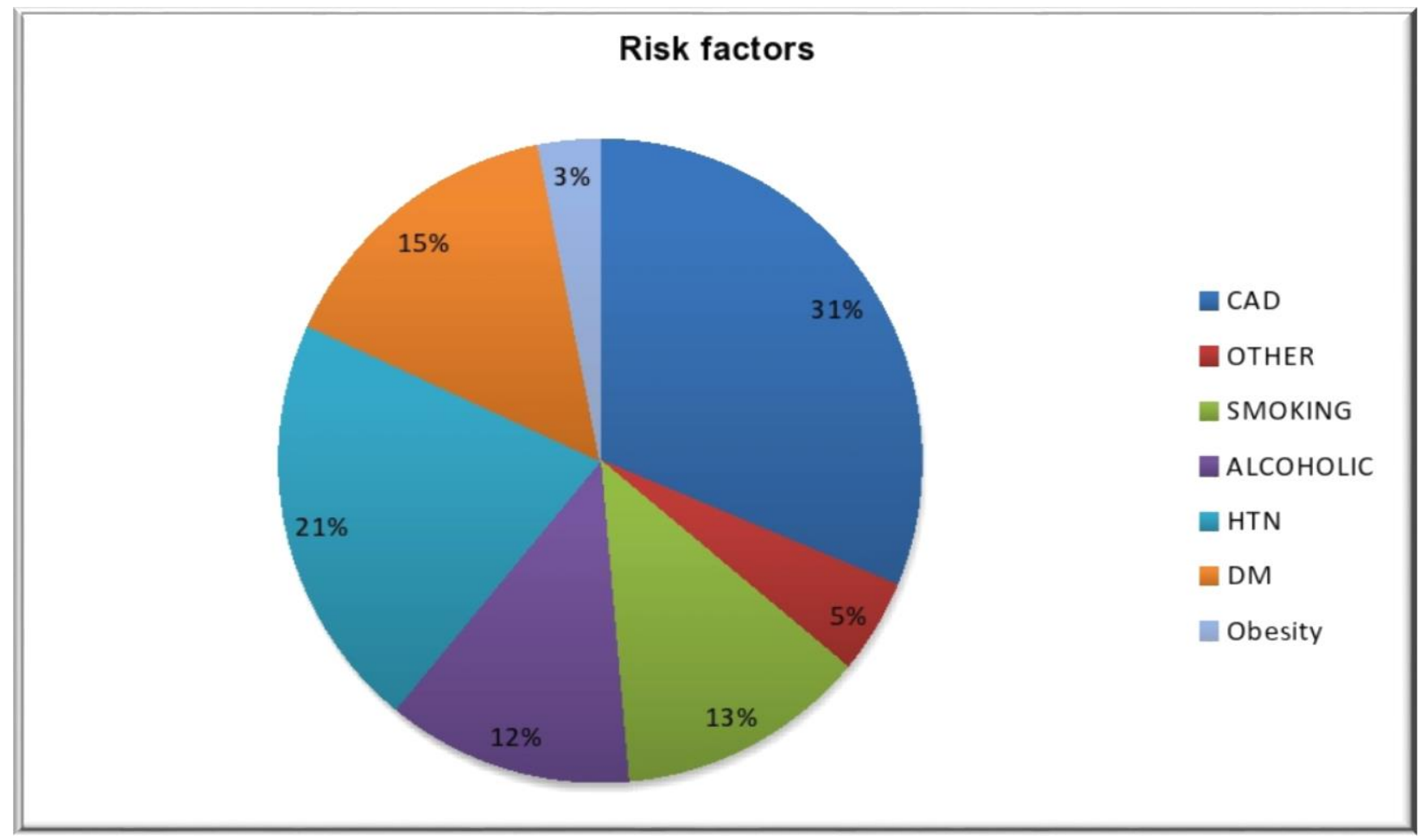

Figure 5: Pictorial representation of different risk factors that cause myocardial infarction

Abbreviations used : Coronary Care Unit (CCU) ; statistical package for social science (SPSS) ; Non-ST Elevated Myocardial Infarction (NSTEMI) ; ST-Elevated Myocardial Infarction(STEMI) ; Cardio Vascular Diseases (CVD) ; myocardial infarction ( MI ) ; Body mass index (BMI) .

\section{CONCLUSION:}

Among different types of modifiable and non-modifiable risk factors, we have analyzed only specific

risk factors which are the major causes for MI such as Hypertension, Diabetes Mellitus, Smoking, Alcohol, any coexisting CVDs, Obesity, and other comorbid conditions. Smoking and Alcohol are preventable risk factors for this disease. Overweight and obese may affect health, and it is necessary to control one's BMI.

Literature was evaluated only on specific individual risk factors, but very small amount in-detail conclusions of such studies were reported by other investigators. In the following study site, it was seen that the highest number of subjects were enrolled for the study belongs to cardiac department. Considering various risk factors, the highest proportion of Coronary Artery Disease was observed followed by hypertension and diabetes. Providing patient education can also help in minimizing the risk factors, thereby reducing the incidence of myocardial infarction. Based on this study, in the following study period it can be concluded that improving the life style of individual can reduce the recurrent chances of myocardial infarction.

Review articles:

1) TuomasKerola et.al., conducted a population based cohort study of "RISK FACTORS ASSOCIATED WITH ATRIOVENTRICULAR BLOCK" was carried out in the MiniFinland Health Survey,during the period between January 1, 1978, to December 31, 1980 where a total of 7217 older than 30 years of age participated in health examinations. The average age of 6146 subjects was 49.2years of which ; 2697 (43.9\%) were men and $3449(56.1 \%)$ were women. The characteristics associated with AV block are Older age $(\mathrm{P}<.001)$, hypertension ( $\mathrm{P}=.002)$, and higher levels of cholesterol $(\mathrm{P}<.001)$, triglycerides $(\mathrm{P}<.001)$, and fasting glucose $(\mathrm{P}<.001)$ were each associated with a higher risk of $\mathrm{AV}$ block. On the whole, this study concludes that the modifiable risk factors like an elevated systolic blood pressure and a higher fasting glucose level were independently associated with AV block ${ }^{6}$.

2) Emily Banks et.al, conducted a prospective study of "TOBACCO SMOKING AND RISK OF 36 CARDIOVASCULAR DISEASE SUBTYPES: FATAL AND NON-FATAL OUTCOMES" in a large prospective Australian study which was carried out from 2006 to 2009 where 267,153 men and women aged 45 were involved in the study. In general, 94.9\% ( $\mathrm{n}=$ 74,141 ) among all current and past smokers reported smoking cigarettes only, $1.8 \%(\mathrm{n}=1379)$ have been smoking cigarettes and cigars or pipes and $1.8 \%(\mathrm{n}=$ 1407) were smoking only cigars or pipes. The attributable fraction was found much higher at younger in comparision to older ages, being $38.2 \%$ in men aged $45-54$ decreasing to $9.3 \%$ in women aged $\geq 75$ years ; it 
was $35.5 \%$ for the age group 45-64. Current tobacco smokers have at least double the risk of developing most significant types of CVD, majorly AMI. Risks of CVD increased with increasing numbers of cigarettes smoked per day and were greatly diminished among those quitting smoking ${ }^{7}$.

3) Sidney $\mathrm{C}$ et.al, conducted a population-based control study on "RISK FACTORS FOR MYOCARDIAL INFARCTION IN LATIN AMERICA" where the 9 risk factors for coronary heart disease - abnormal lipid levels, smoking, hypertension, diabetes, abdominal obesity, psychosocial stress, regular physical activity, and consumption of fruits, vegetables, and alcohol - accounts for $90 \%$ or more of the PAR for both men and women worldwide. The PAR for abdominal obesity in the Latin American countries studied was $48.5 \%$, followed by $40.8 \%$ for dyslipidemia and $38.4 \%$ for smoking. In their study, few major gender differences were noted, where men more likely to smoke than women $(54 \%$ versus $12 \%$ ) and women are more likely to have abdominal obesity (35\% versus $9 \%$ ). Intra-abdominal fat has been reported to be independently correlated with the metabolic syndrome, where the patients have twice the risk of developing cardiovascular disease and a 4 times the risk for diabetes ${ }^{8}$.

4) Saif Al-Shamsi et.al, conducted a retrospective cohort study of "INCIDENCE OF CARDIOVASCULAR DISEASE AND ITS ASSOCIATED RISK FACTORS IN AT-RISK MEN AND WOMEN IN THE UNITED ARAB EMIRATES: A 9- YEAR RETROSPECTIVE COHORT STUDY" at Tawam Hospital, Al-Ain, during the period of April 1, 2008, and December 31, 2008 where 977 subjects are involved. In both males and females, a low eGFR was a significant risk factor for major CVD. The SBP was a strong predictor of major CVD, where the risk increases by $20 \%$ and $58 \%$ for every $10 \mathrm{mmHg}$ SBP increase in men and women, respectively. The major CVD risk increased by $16 \%$ in men and $24 \%$ in women for each $1 \%$ rise in the HbA1c level. In this study, they have found that the incidence rate of major CVD was majorly in men than in women. Cigarette smoking influences other cardiovascular risk factors, for example, DM and serum lipids and it has a multiplicative association with HTN on incident CVD 9 .

\section{REFERENCES}

[1] Gupta R, Mohan I, Narula J. Trends in coronary heart disease epidemiology in India. Annals of global health. 2016 Mar 1;82(2):307-15.

[2] Jayaraj JC, Davatyan K, Subramanian SS, Priya J. Epidemiology of Myocardial Infarction. InMyocardial Infarction 2018 Nov 5. IntechOpen.

[3] Lilly LS, Braunwald E. Braunwald's heart disease: a textbook of cardiovascular medicine. Elsevier Health Sciences; 2012.

[4] Benjamin EJ, Virani SS, Callaway CW, Chamberlain AM, Chang AR, Cheng S, Chiuve SE, Cushman M, Delling FN, Deo R, de Ferranti SD. Heart disease and stroke statistics-2018 update: a report from the American Heart Association. Circulation. 2018 Mar 20;137(12):e67.

[5] Benjamin EJ, Muntner P, Bittencourt MS. Heart disease and stroke statistics2019 update: a report from the American Heart Association. Circulation. 2019;139(10):e56-28.

[6] Kerola T, Eranti A, Aro AL, Haukilahti MA, Holkeri A, Junttila MJ, Kenttä TV, Rissanen H, Vittinghoff E, Knekt P, Heliövaara M. Risk factors associated with atrioventricular block. JAMA network open. 2019 May 3;2(5):e194176-.

[7] Banks E, Joshy G, Korda RJ, Stavreski B, Soga K, Egger S, Day C, Clarke NE, Lewington S, Lopez AD. Tobacco smoking and risk of 36 cardiovascular disease subtypes: fatal and non-fatal outcomes in a large prospective Australian study. BMC medicine. 2019 Dec 1;17(1):128.

[8] Smith Jr SC. Risk factors for myocardial infarction in Latin America: sobrepeso y obesidad.

[9] Al-Shamsi S, Regmi D, Govender RD. Incidence of cardiovascular disease and its associated risk factors in at-risk men and women in the United Arab Emirates: a 9-year retrospective cohort study. BMC cardiovascular disorders. 2019 Dec 1;19(1):148.

\section{AUTHORS}

First Author - Anju Sharma, Pharm D, Malla Reddy Institute of Pharmaceutical Sciences, Dhulapally, Hyderabad, 500014, India, sharmaanju253@gmail.com

Second Author - Konda. Sushritha, Pharm D, Malla Reddy Institute of Pharmaceutical Sciences, Dhulapally, Hyderabad, 500014, India, sushritha22496@gmail.com

Third Author - Konda. Suman, Pharm D, Malla Reddy Institute of Pharmaceutical Sciences, Dhulapally, Hyderabad, 500014, India, sumankonda282@gmail.com

Fourth Author - Palakurthy. Harish Goud, Pharm D, Malla Reddy Institute of Pharmaceutical Sciences, Dhulapally, Hyderabad, 500014, India, harishgoud@gmail.com Fifth Author - Sadanandamn Akari, Pharm D, Malla Reddy Institute of Pharmaceutical Sciences, Dhulapally, Hyderabad, 500014, India, sadhapharmd@gmail.com 